\title{
DIONYSOS À NEW YORK : LES FRAGMENTS DU MYTHE DIONYSIAQUE DANS ONYSOS LE FURIEUX DE LAURENT GAUDÉ
}

Onysos le furieux est le premier texte théâtral de l'écrivain contemporain français Laurent Gaudé. Écrit en 1997 et publié en 2000, ce monologue au souffle épique propose une nouvelle lecture du mythe dionysiaque. Assis sur le quai d'un métro newyorkais, Onysos prend la parole et nous emmène dans un voyage à travers le temps et à travers son histoire, qui est, en réalité, celle de Dionysos - un peu moins dieu, un peu plus homme. Le dessein de l'écrivain n'est pas de réécrire ou d'adapter une des nombreuses variantes classiques du mythe de Dionysos, mais de tisser un nouvel espace mythologique, sa propre antiquité imaginaire, à partir de particules mythiques hétérogènes et amalgamées. En nous appuyant sur la mythocritique de Philippe Walter, nous analyserons dans ce travail les mythèmes, les plus petites unités de discours significatives sur le plan mythique, qui constituent le puzzle du mythe dans Onysos le furieux. Nous tâcherons de retracer les mythèmes empruntés aux différentes variantes classiques du mythe de Dionysos (la naissance tragique, le démembrement, la descente aux Enfers, l'épisode avec Proscumnos, Dionysos travesti, etc), ainsi que les mythèmes construits, auctoriaux.

Mots clés : mythe dionysiaque, Onysos le furieux, mythèmes, mythocritique, Laurent Gaudé, littérature française contemporaine.

\section{INTRODUCTION}

Dramaturge, romancier et poète, Laurent Gaudé est né à Paris le 6 juillet 1972. Après ses études en Lettres Modernes et Études Théâtrales à l'Université Paris III, il publie sa première pièce de théâtre Onysos le Furieux (1997), puis ses œuvres dramatiques Pluie de cendres (1998) et Combat de Possédés (1999). En 2001, Gaudé publie son premier roman Cris et la pièce Cendres sur les mains. Deux ans plus tard, il obtient le Prix Goncourt des Lycéens pour son roman La Mort du Roi Tsongor (publié en 2002). En 2004, il

\footnotetext{
*nebojsa.vlaskalic@ff.uns.ac.rs
} 
publie son roman Le Soleil des Scorta pour lequel il obtient le Prix Goncourt la même année (le roman a été traduit dans 34 pays). Jusqu'à présent, Laurent Gaudé a publié 11 romans, 17 pièces de théâtre, 2 recueils de nouvelles et 2 recueils de poésie ${ }^{1}$.

Tout comme la production littéraire de l'extrême contemporain, l'œuvre littéraire gaudéenne réinterroge sans cesse le présent par un regard rétrospectif. Cette interrogation du passé se présente comme « une exploration archéologique de nos savoirs et généalogique de notre identité. [...] Il s'agit parfois de comprendre qui nous sommes à la lumière de ce que nous étions » (Rubino, 2006: 10). Dans un monde qui change "il devient important de réactualiser les mythes pour qu'ils continuent à parler aux êtres humains, s'agissant de les aider à trouver leurs repères et leurs valeurs " (Moingeon, 2007 : 12). Puisque les écrivains contemporains «se placent dans la position d'héritiers d'un imaginaire mythique et proposent sa reconfiguration qui représente un point de basculement culturel à cet égard » (Heidmann, 2010 : 55), le rôle de l'écrivain s'affirme fondamental dans le recours au mythe dans la littérature d'aujourd'hui.

\section{LE CADRE THÉORIQUE - MYTHOCRITIQUE ET MYTHÈMES}

La mythocritique est un courant critique qui étudie les mythes dans un contexte littéraire. Le terme mythocritique a été forgé par Gilbert Durand (1992), qui décrit la mythologie comme l'application d'un objet à un autre objet, plus précisément, l'analyse d'un récit par le biais d'un autre récit. Cette étude des textes dans l'optique du mythe pose que " tout 'récit' entretient une relation étroite avec le sermo mythicus, le mythe » (Durand, 1996 : 230). Pierre Brunel (1992) reprend la thèse de Durand et

esquisse les fondements d'une mythocritique dont le but est d'étudier systématiquement la présence du mythe dans le texte littéraire. Le mythe est défini par ses trois fonctions: il raconte, il explique, il rélève. [...] Une

\footnotetext{
${ }^{1}$ Jusqu'à présent, seulement deux de ses romans ont été traduits en Serbie : La Mort du roi Tsongor (en 2004, traduction de Mirjana Vukmirović, édité par la maison d'édition Filip Višnjić) et Le Soleil des Scorta (en 2005, traduit par Mirjana Vukmirović, édité par PAIDEIA).
} 
démarche analytique permet le découpage d'un récit mythique en séquences d'un scénario (Leonardy, 1994 : 5-6).

Dans la tentative de définir la mythocritique dans l'époque contemporaine, Danièle Chauvin et Philippe Walter écrivent dans la préface de Questions de mythocritique, Dictionnaire : « le postulat de la mythocritique est de tenir pour essentiellement signifiant tout élément mythique, patent ou latent, et donc d'organiser à partir de lui toute l'analyse de l'œuvre » (Chauvin, Walter, 2005: 7). La mythocritique identifie un mythe à partir de ses mythèmes, "les plus petites unités de discours mythiquement significatives » (Durand, 1992 : 343), qui peuvent former un épisode, un motif, un thème ou un personnage dans le récit mythique. Walter voit le mythe comme un puzzle des mythèmes épars qui le constituent :

Analyser un mythe, ce n'est pas s'intéresser à des motifs narratifs isolés [...]. C'est plutôt découvrir dans les textes des groupes des motifs liés entre eux et relevant d'une séquence narrative particulière. La récurrence de ces motifs et de ces séquences dans un même texte ou dans des textes distinctifs fait émerger le puzzle du mythe. [...] Le mythe est donc un récit, mais ce récit n'a pas de forme fixée et rigide. Ses motifs constituants peuvent connaître des variations réglées qui ne remettent pas en cause le développement narratif de la séquence de base (Walter, $2008: 21$ ).

En nous appuyant sur la mythocritique walterienne, nous tâcherons de retracer les mythèmes dans l'œuvre Onysos le furieux de Laurent Gaudé. Tout en suivant la verticalité dans laquelle l'écrivain français contemporain essaie de réunir l'antiquité et la modernité, nous individuerons les mythèmes que l'auteur emprunte au mythe antique dionysiaque, mais qui sont transposés dans un contexte différent dans sa pièce. Dans la tentative de comprendre quelle est la fonction du mythe dionysiaque dans Onysos le furieux, outre les mythèmes empruntés et hybrides, nous analyserons aussi les mythèmes construits par l'auteur.

\section{L'INSPIRATION MYTHIQUE DANS L'OEUVRE LITTÉRAIRE DE LAURENT GAUDÉ}

Poussé par son désir de comprendre, expliquer et décrire le monde contemporain, Laurent Gaudé recourt aux mythes, tandis qu'il fait de l'Histoire un cadre, une mise en scène, ce qui lui permet de placer dans la fiction ses contemplations modernes amalgamées avec ses inspirations classiques. Auteur 
d'innovations et libres variations, comme le caractérise Dominique Viart (2013: 206-207), il voit le mythe comme une réalité vivante et multiforme qui aide à saisir des forces immanentes à l'homme. Le mythe, explique Gaudé, est une verticalité entre l'antiquité et modernité qui invite à explorer l'homme dans sa diversité. Tant que le mythe est porteur d'énigmes, de choses inconfortables et non-résolues, il porte aussi une sagesse, un soulagement, celui que les hommes ont été depuis toujours devant les mêmes grandes questions existentielles. Les mythes continuent à servir à la compréhension de l'homme et le mettent face à une vérité terrible ${ }^{2}$. Dans une optique similaire, Jean-Pierre Vernant ${ }^{3}$ définit le mythe comme "le récit [qui] a pour fonction d'apporter une réponse à des problèmes fondamentaux, comme le statut des dieux, l'existence de la mort, la condition des hommes, les formes de la vie sociale » (Vernant, $1980: 21-25$ ).

Bien que Gaudé insiste sur le fait qu'il n'a jamais considéré son travail ${ }^{4}$ comme un renouvellement du mythe ou une déclinaison littéraire des mythes anciens, l'écrivain dialogue incessamment avec les textes issus des mythologies différentes. L'intention de l'auteur n'est pas de reprendre des mythes bien connus, mais de construire - en utilisant des particules mythiques - un nouvel espace mythologique, dans lequel il entremêle des mythèmes hétérogènes (originaux et/ou empruntés à des mythologies diverses). Comme le souligne Augé-Rabier :

Gaudé ne réécrit pas les mythes au sens propre du terme, il extrait leurs parfums qu'il combine à d'autres essences. [...] Cette réécriture ne s'inscrit pas dans la mouvance des réécritures des $\mathrm{XX}^{\mathrm{e}}$ et $\mathrm{XXI}^{\mathrm{e}}$ siècles : elle n'est ni retour aux sources [...], ni revendication politique. Le mythe (chez Gaudé) prend un nouvel envol (Augé-Rabier, 2013, para. 2).

${ }^{2}$ L'écrivain nous a donné sa définition du mythe durant notre entrevue exclusive à Rome, le 21 novembre 2015.

3 Parmi d'autres auteurs qui ont probablement influencé l'intérêt de Gaudé pour la mythologie grecque, il faut mentionner Pierre Vidal-Naquet (v. Mythe et Tragédie en Grèce ancienne (2005)) et Marcel Detienne (v. L'Invention de la mythologie (1981), Dionysos mis à mort (1996), etc).

${ }^{4}$ Nous pourrions partager le corpus littéraire gaudéen recourant au mythe en quatre groupes : les mythes d'inspiration classique (entre autres : Orphée, Dionysos), les mythes de diverses inspirations (Eldorado, le mythe du Golem, etc.), les mythes construits (auctoriaux) et les mythes croisés (Médée Kali). 
Laurent Gaudé ne convoque pas les mythes pour revenir à l'antiquité, mais pour mettre en lumière la condition des hommes modernes. Parfois un prétexte dramatique ou un exercice de style, le mythe dans l'œuvre gaudéenne est un mythe effondré, émietté et disséminé qui plane partout dans le texte pour amplifier sa dimension tragique et poétique.

\section{ONYSOS LE FURIEUX}

Gaudé écrit le texte qui marquera le début de sa carrière au début de printemps de 1996. En 1997, à l'âge de 25 ans, le jeune écrivain envoie par la poste l'histoire du héros épique, Onysos le furieux, à Hubert Gignoux ${ }^{5}$, ancien acteur et metteur en scène, qui était également directeur du Théâtre National de Strasbourg. En septembre 1997, Gignoux lit la pièce sur les ondes de France Culture dans l'émission de Lucien Attoun ${ }^{6}$, qui, attiré par le texte de Gaudé, se décide à le publier en tapuscrit la même année7. Onysos le furieux sera édité par Actes Sud en 1999 et joué pour la première fois en 2000 au Théâtre National de Strasbourg dans une mise en scène de Yannis Kokkos, avec Jean-Yves Dubois ${ }^{8}$.

Écrit sous la forme d'un long poème en prose au souffle épique, le premier texte de Gaudé est un monologue qui met au centre de la narration Onysos, un vieillard fangeux et ridé qui s'adresse à un personnage inconnu, assis à côté de lui sur le quai d'un métro new-yorkais. En contemplant son

${ }^{5}$ Gignoux, qui a curieusement découvert et aidé Bernard-Marie Koltès, s'intéressait aux textes de théâtre contemporain et a décidé d'aider le jeune Gaudé à publier sa première pièce de théâtre.

${ }^{6}$ Attoun était également fondateur et directeur du Théâtre Ouvert avec sa femme Micheline. Le Théâtre Ouvert est un théâtre d'essais et de création dont la mission est de découvrir de nouveaux textes d'écriture contemporaine. Ce théâtre, né en 1971 au Festival d'Avignon et installé à Paris en 1981, est situé aujourd'hui non loin de Pigalle, dans le $18^{\mathrm{e}}$ arrondissement à Paris.

${ }^{7}$ Simultanément avec la publication de ses pièces en tapuscrit, le jeune Gaudé envoie des textes brefs pour la rubrique consacrée aux textes inédits de nouveaux auteurs dans l'ancienne revue théâtrale Du Théâtre, la revue des éditions Actes Sud. Claire David, la responsable pour le théâtre, commence à les publier, décidant de faire de Gaudé un auteur de la maison d'édition renommée.

${ }^{8}$ La pièce sera mise en scène de nouveau en France en 2007 par Charlie Brozzoni à Bonlieu Scène nationale d'Annecy, avec Carlo Brandt. Une mise en scène anglaise de Severine Ruset aura lieu au Theatre 503 à Londres en 2005 (et reprise durant le Festival d'Edimbourg 2006 au Traverse Theatre), avec Chris Porter. 
« camarade » muet, le protagoniste prend la parole après « milliers d'années de silence » et lui raconte, pendant une nuit, sa vie pleine de cruautés et de passions. Au fur et à mesure de son récit, sa voix rêche et criarde commence à "charmer d'une douceur», les milles rides qui couvrent son visage disparaissent et sa peau noire, rugueuse et vieille rajeunit. Divisée en six chants et deux prologues, l'histoire d'Onysos se divise aussi entre deux temporalités $^{9}$ : en premier lieu, celle de la vie précédente du héros dans l'antiquité (elle-même divisée en multiples épisodes), et celle de sa vie dans le New York d'aujourd'hui. Oublié par le monde et épuisé après une vie de folie et de destruction - Onysos habite New York, la mégapole moderne qu'il a choisie parce qu'elle lui ressemble et grouille comme lui :

Est-ce que mon visage et ma voix te font peur?

La vieillesse de ma peau te répugne-t-elle?

Ne t'inquiète pas, je rajeunirai. [...]

Ne t'inquiète pas, camarade, je vais tout te raconter.

J'ai enfin trouvé la cité dont je suis.

Je suis né d'abord, j'ai erré à la recherche d'un endroit à ma taille, et je ne l'ai trouvé que maintenant, très longtemps après, à l'instant presque de mourir.

Mais maintenant je sais, et je peux dire que je suis né pour New York (Gaudé, $2000: 10)$.

L'histoire d'Onysos est, en réalité, celle de Dionysos. Dans la mythologie grecque, Dionysos est le dieu du vin, de la vigne, de l'extase et de l'immodération. Éternellement changeant et libéré de toutes les contraintes morales et matérielles, Dionysos est un personnage complexe, de ce qui témoignent de nombreuses variantes du mythe dionysiaque :

De tous les dieux de l'Olympe, Dionysos est celui dont la figure est la plus difficile à cerner. Qu'on examine ses origines, les épisodes de son enfance, son aspect physique, son caractère, sa place dans la cité, l'utilisation symbolique qui en a été faite au cours de l'Histoire, ou les interprétations des mythologues, le dieu nous échappe. Riche, complexe, fuyant, il est le dieu des métamorphoses. Il est l'insaisissable (Brunel, 2000 : 443).

${ }^{9}$ Tandis que les deux prologues de la pièce sont situés dans le monde moderne, les six chants sont construits sur les passages fréquents entre les deux temporalités différentes. L'une des particularités de ce texte est le manque de didascalies. 
Pour mettre la dimension divine du personnage mythique au second plan, Gaudé efface le préfixe $d i$ - du nom du héros antique et crée ici Onysos, un « fort mauvais dieu qui saigne et hurle comme les hommes, qui bande et rit comme les hommes » (Gaudé, 2000: 40). Outre le nom abrégé, l'auteur met en évidence le caractère de son héros en utilisant l'épithète furieux, ce qui est une référence au caractère du dieu antique, caractérisé par sa fureur (plus notamment chez Homère). Après une vie pleine de monstruosités, mais aussi de souffrances, ce dieu - plus humain que divin - a choisi de vivre à New York, aux marges de la société moderne, où il protège les humiliés.

Même si l'action du drame ne dure qu'une nuit, elle est partagée entre un passé mythique et une époque qui semble être assez récente. La pièce est construite sur un changement constant du rythme de la narration : grâce à l'oralité puissante et suggestive du héros principal, on passe en un clin d'œil de New York à l'antiquité, pour revenir toujours sur le quai du métro new-yorkais. L'auteur ne le reconnaît pas, mais la pièce Onysos le furieux propose une nouvelle lecture du mythe dionysiaque dans une optique contemporaine. Pleine de références aux nombreuses variantes du mythe dionysiaque, la pièce unit de nombreux mythèmes (empruntés, hybrides et construits par l'auteur) que nous analyserons dans le chapitre suivant de ce travail.

\section{LES FRAGMENTS DU MYTHE DIONYSIAQUE - LES MYTHÈMES DANS ONYSOS LE FURIEUX}

Même s'il déclare qu'il ne veut pas adapter une des nombreuses variantes du mythe dionysiaque, il faut dire, tout de même, que l'écrivain dialogue incessamment avec de nombreux textes mythologiques, comme Les Bacchantes d'Euripide et Métamorphoses d'Ovide, auxquels il ajoute de nouveaux fragments de l'histoire méconnue de Dionysos. Le changement du nom du héros et son déplacement dans un monde entièrement différent, lui servent de prétexte pour se libérer du fardeau des nombreuses versions connues du mythe antique et ainsi explorer l'humanité du personnage mythique. Pour accentuer l'humanité du dieu grec, Gaudé reprend et 
transforme certains mythèmes de l'antiquité, mais aussi en en écrivant luimême quelques-uns pour offrir une nouvelle lecture du mythe antique ${ }^{10}$.

Au-delà des mythèmes de la création par le maître des dieux et celui du démembrement empruntés au mythe antique dionysiaque, l'auteur recontextualise d'autres fragments liés à la naissance de Dionysos dans son texte. Bien que la (re)naissance violente et le démembrement du nouveau-né Dionysos se présentent dans plusieurs versions ${ }^{11}$, dans le texte gaudéen, Onysos naît à Tepe Sarabe, dans la poussière des monts Zagros. À peine sorti du ventre de sa mère Ino, le nouveau-né Onysos a été tué par un groupe d'hommes ${ }^{12}$. Après l'avoir démembré, les hommes étranges rassemblent les morceaux de sa chair et les font bouillir dans un chaudron de cuivre, mais ils oublient le cœur :

Je suis celui qui n'est pas né d'une femme.

Certes Ino m'a tenu dans son ventre et Ino a poussé pour que je sorte d'entre ses cuisses,

Mais mon père est le maître des dieux et il n'était pas besoin d'une femme pour que je sois projeté dans la poussière des monts Zagros [...]

Il y a eu une grande fête. [...]

Un groupe d'hommes et là qui ne danse pas. [...]

10 On retrouve la même amalgamation des mythèmes dans la pièce de théâtre Médée Kali, dans laquelle l'auteur raconte l'histoire inconnue de Médée, en reliant trois figures mythiques et deux mythologies - grecque et indienne. Pour plus de détails voir Vlaškalić, N. (2020). Mitska slagalica: ukršteni mitovi u delu Medeja Kali Lorana Godea. Jezici i kulture u vremenu i prostoru, IX/2, 39-47.

${ }^{11}$ Brunel en met en lumière quelques-unes. Après avoir été arraché au ventre de sa mère Sémélé, Dionysos est introduit dans la cuisse de Zeus, de laquelle il naîtra une deuxième fois: « De là vient l'une des étymologies de son nom : né deux fois » (Brunel, 2000 : 445). Dans une autre version, le dieu Hermès confie l'enfant, sur l'ordre de Zeus, au roi d'Orchomène, Athamas, et à son épouse Ino, qui revêt le petit Dionysos d'habits féminins, pour le protéger de la jalousie d'Héra. Dans l'épisode crétois de DionysosZagreus (le nom Zagreus apparaît au VIème siècle avant J.-C.), Dionysos a de nouveaux parents nourriciers - les Courètes - qui, dansant autour de l'enfant, ne s'aperçoivent pas que les Titans tuent le petit, le démembrent et font cuire ses morceaux dans un chaudron (tous sauf le cœur). Jacques Lacarrière (1998: 436) illustre deux versions de la naissance étrange de Dionysos - la version dans laquelle Dionysos est né de la cuisse de Zeus, et celle, plus ancienne, de la naissance du dieu Zagreus, qui passait pour la première incarnation de Dionysos.

12 Les Titans qui tuent Dionysos sont, eux aussi, humanisés chez Gaudé et deviennent ici un groupe d'hommes étrangers qui tuent Onysos. 
Ils m'entourent maintenant [...]

Ils me saisissent et tirent chacune de mes extremités. [...]

Egorgé, déchiré et démembré, le petit Onysos est né.

Mais les imbéciles, dans leur démence, ont oublié le cœur.

Et à partir du cœur, mon père, parce qu'il est le maître des dieux,

Parce que lui a entendu les cris que je n'ai pas poussés,

De ses mains d'argile m'a fait naître à nouveau (Gaudé, 2000 : 11-13).

La nourricière de Dionysos, Ino, chez Gaudé devient la mère d'Onysos. Même s'il ne décrit pas en détail la relation entre la mère et son fils, ce rapport donne à Gaudé une excellente opportunité d'illustrer la sensibilité de son protagoniste. Au fil de ses errances, Onysos pleure car il pressent que sa mère est morte ${ }^{13}$. Profondément triste, Onysos rencontre sur la route Proscumnos ${ }^{14}$, «le sodomite, ivrogne superbe, voleur furieux et ignoble bonhomme » qui l'aidera à descendre en Hadès pour retrouver sa mère morte, à condition qu'il lui accorde ses faveurs à son retour ${ }^{15}$. Après la scène touchante où le demi-dieu pleure à la vue de sa mère morte et muette aux Enfers ${ }^{16}$, Onysos remonte sur terre, où il trouve Proscumnos mort "de maladie, de fièvre ou de solitude", mais il remplit sa part de la promesse. En changeant et en mêlant trois

\footnotetext{
13 « Une peine immense m'étreignait que je ne distinguais pas et qui me pesait. Je pensais à Ino.

Mère chérie, Je n'ai connu ta présence que quelques heures, Je me souviens à peine des traits de ton visage Mais le grain de ta voix résonne à jamais dans mon cœur. Ino, Ce soir, sans que je sache d'où cela me vient, la certitude de ta mort me fait verser de grosses larmes océanes. [...]
}

Sur la route de Ur, la tristesse qui m'étreint m'annonce que tu es morte » (Gaudé, 2000 : 22-23).

${ }^{14}$ Le personnage de Prosumnos, que l'on retrouve dans une version du mythe antique dionysiaque, dans la pièce gaudéenne devient Proscumnos.

15 « Proscumnos connaissait le chemin du pays des Morts.

Il m'assura qu'il savait où était l'entrée et comment faire, une fois dans le labyrinthe pour ne pas se perdre.

Nous avons fait un pacte.

Il me livrait son secret et je devais, une fois revenu, lui accorder mes faveurs.

Proscumnos le sodomite me proposa son marché et j'acceptai » (Gaudé, $2000: 24$ ).

16 «J'ai retrouvé Ino mais elle n'a pas pu parler et mes yeux pleuraient tellement que je n’ai pas distingué son visage. » (Gaudé, $2000: 24$ ). 
mythèmes empruntés aux textes dionysiaques de l'antiquité - celui de la descente de Dionysos aux Enfers, celui de sa promesse tenue à Pros(c)umnos ${ }^{17}$ et celui du figuier à l'entrée de l'Hadès, l'écrivain crée ici un exemple marquant du puzzle du mythe qui lui sert à dévoiler les caractéristiques humaines d'Onysos : sa fidélité, sa sensibilité et sa sexualité ambiguë.

Gaudé développe le sujet de l'exagération du plaisir sexuel dionysiaque : libéré de toutes les contraintes, Onysos est, juste comme Dionysos, voluptueux et sexuellement libre. Outre le mythème de rites orgiastiques avec son armée des femmes - la horde de furies, c'est-à-dire les nouvelles Bacchantes, dans la pièce gaudéenne nous retrouvons aussi le (Di)Onysos travesti ${ }^{18}$, un autre mythème notable emprunté au mythe antique. La sexualité ambivalente d' Onysos est abordée aussi dans l'épisode du crime de Penthée. Curieux de participer aux orgies d'Onysos sur les collines qui surplombent le Nil, Penthée, le jeune roi africain, se travestit en femme et se mêle à la horde des femmes. Enragé, puisqu'il le prend pour une moquerie, Onysos viole Penthée travesti et lui confie à ses furies pour qu'elles le tuent. Gaudé reprend cette histoire du mythe antique dionysiaque, mais il le transforme en le transposant dans un contexte africain. Les ressemblances intertextuelles avec Les Bacchantes d'Euripide et Métamorphoses d'Ovide sont évidentes : l'auteur réinterprète les mythèmes du rite orgiastique, du travestissement de Penthée et de son démembrement par les furies. Cependant, en transformant le lieu du récit, l'écrivain crée ici un mythème hybride : Penthée n'est plus le roi de Thèbes,

17 Un exemple lumineux de l'intertextualité, la scène de la rencontre entre Onysos et Proscumnos et de leur serment est, en fait, la réécriture d'un récit que Brunel mentionne dans son Dictionnaire des mythes littéraires, à propos de la sexualité de Dionysos: « Même dans ses amours Dionysos demeure l'insaisissable. Un curieux récit, dont la version la plus développée se trouve chez Clément d'Alexandrie (Protreptique, II, 34, 3-5), fait de l'éraste hétérosexuel d'Ariane l'éromène homosexuel de Prosumnos. Contre promesse de lui accorder ses faveurs à son retour, Dionysos obtient de Prosumnos les indications pour descendre aux Enfers. Mais quand il revient, Prosumnos est mort. Alors Dionysos se rend à son tombeau : 'Il coupe, au hasard, une branche de figuier, lui donne la forme d'un membre viril, et s'en sert pour remplir, à l'égard du mort, sa promesse' » (Brunel, 2000 : 448). Bien que Gaudé intègre tout l'épisode mythique de la promesse tenue dans son récit, il change le prénom de Prosumnos et recontextualise le mythème du figuier sodomite.

18 Onysos se travestit deux fois dans la pièce : quand il conquiert la ville de Tepe Moussian et aux funérailles de sa bien-aimée Séléna. 
mais un jeune roi africain « encore beau car aucune bataille, à ce jour, ne l'avait meurtri dans sa chair » qui règne sur Akhetanon, royaume inventé par l'auteur, au bord du Nil. À la poursuite incessante d'un troisième genre hybride, qui essaierait de marier l'épique, le lyrique et le tragique, Gaudé crée ici l'atmosphère de l'affabulation renvoyant à une tragédie antique mêlée à une épopée ou un conte africain ${ }^{19}$.

Un des exemples les plus marquants de l'humanité d'Onysos que Gaudé met en lumière est certainement l'épisode de la chute de Troie. En mêlant l'histoire de la guerre troyenne à la naissance de la tragédie grecque, l'écrivain décrit le dieu qui pleure en se battant ${ }^{20}$ avec les hommes qui perdent. La veille de la chute de Troie, pour atténuer la douleur de ceux qui allaient perdre la vie et ainsi adoucir leur mort, Onysos invente le théâtre. Touché par leur stoïcisme et leur tristesse, Onysos décide de rester auprès les Troyens dans leur bataille déjà perdue.

Au-delà des mythèmes empruntés puis transformés, l'écrivain écrit deux nouveaux épisodes de l'histoire mythique pour explorer plus en détail l'humanité du mi-homme, mi-dieu enragé. Le mythème crucial pour la compréhension de la vulnérabilité du demi-dieu est l'épisode inventé à Akko, en Phénicie, où Onysos trouve l'amour ${ }^{21}$ avec la princesse Séléna, la fille du roi Marador. Pour pouvoir faire l'amour avec Séléna sereinement, Onysos met feu aux ailes du palais, tue les hommes qui le gênent et détruit la ville d'Akko. Scandalisé par ces accouplements contre nature, Madador tue sa fille, tandis qu'Onysos réussit à s'enfuir grâce à sa "rage de l'homme et rage du bouc ». Transformé en chien, Onysos court à nouveau en maudissant la ville dans laquelle il reviendra travesti en vielle femme aux funérailles de Séléna pour se venger. Après les funérailles de sa bien-aimée, Onysos s'enfuit sur une barque, où il commence à vieillir, seul et misérable :

Calciné par les feux du deuil.

C'est sur cette barque que j'ai commencé à vieillir.

19 On peut trouver le même procédé narratif dans le roman La Mort du roi Tsongor ainsi que dans la pièce de théâtre Salina.

20 «J'ai lutté moi aussi. [...] Et moi aussi je pleurais en me battant, je pleurais de voir autour de moi le sol jonché des corps de ceux que j'aimais » (Gaudé, $2000: 43$ ).

21 «Je n'avais jamais pensé qu'il me serait donné de connaître l'emprise d'une femme sur mes sens » (Gaudé, $2000: 33$ ). 
C'est sur cette barque que mon visage a connu ses premières fatigues.

Et si je rajeunis maintenant, au fur et à mesure que je te parle, s'il est vrai que lorsque j'aurais fini mon histoire mes mains ne trembleront plus, S'il est vrai que ma tête déjà a cessé de dodeliner, Je sais bien que ces premières rides, Nées lorsque Akko brûlait, Jamais ne disparaîtront.

Je dois être un fort mauvais dieu qui chavire et se ride (Gaudé, 2000 : 39-40).

Dans l'épisode inventé, Onysos l'immortel, le demi-dieu magnétique et féroce, vieillit après la perte tragique de son amante. Ses grandes rides d'Akko, ces "isthmes de tristesse qui découpent [son] visage en plusieurs continents » Onysos gardera pour toujours, comme le «souvenir tailladé de Séléna ». Même si dans cet épisode l'auteur développe et unit d'autres mythèmes, comme ceux du magnétisme dionysiaque et du dieu de la vigne ${ }^{22}$, il construit le récit autour de la sensibilité du héros, impuissant devant le destin et la mort, ce qui donne une valeur poétique particulière à ce mythème et au texte entier.

Le deuxième mythème auctorial que nous étudierons est l'épisode de la vie d'Onysos à New York. Après une vie d'errances et cruautés, à la recherche d'un endroit à sa taille, Onysos choisit, en dépit de son mépris pour les villes, de ne pas détruire New York et de s'installer dans la mégalopole qui lui ressemble. Dans cette mégalopole "qui a été construit pour lui », Onysos attend le jour de sa vengeance où il va se rappeler à la mémoire des hommes. Cependant, c'est à New York que le dieu insaisissable subit sa métamorphose la plus importante: il devient plus homme que jamais. En ressentant de la sympathie pour les hommes qui n'ont rien et qui ne sont rien, Onysos, furieux à cause des inégalités entre les hommes, décide de protéger en secret les marginaux de la société moderne. Comme cela, Gaudé écrit l'histoire méconnue

22 Le mythème du dieu de la vigne, motif emprunté au mythe antique, est aussi recontextualisé dans le texte gaudéen pour illustrer le côté humain du héros. Onysos plante des vignes après ses crimes, excès sexuels ou après certains regrets et blessures de l'âme : sur le lieu du crime de Penthée, Onysos plantera des vignes sauvages qui donneront du vin acide, carnivore ; pour entrer dans la chambre de Séléna, Onysos fera pousser une vigne vierge, la même vigne qui lui servit d'échelle à Troie. Il y a aussi le figuier sodomite, le symbole de la promesse tenue à Proscumnos, ainsi que les branches de myrthe avec lesquelles les femmes d'Akko se masturbent sur les places et les avenues, prises de fièvre folle causée par Onysos. 
du dieu à la peau d'ébène qui, comme un saint protecteur, veille sur ses frères et sœurs humiliés - les nègres, les drogués, les prostituées et les émigrés :

Regarde New York, regarde tous ces visages.

Les émigrés ont faim, grelottent de froid et de frustration.

Je vais me fondre dans cette ville que j'aime

Parce que New York a été construite pour moi.

Je vais me mêler à tous ces nègres et ces négresses, tous ces parias

que la ville essaie de cacher sans y parvenir,

Parce que j'ai en haine ces petits Blancs qui se blottissent bourgeoisement les uns contre les autres dans des quartiers où les avenues sont propres et ombragées.

J'ai moi aussi la peau d'ébène. [...]

Je pourrais te dire que je n'ai jamais été aussi fort qu'aujourd'hui, que mes compagnons n'ont jamais été aussi nombreux. [...]

Je pourrais te dire que je suis aujourd'hui le plus puissant des dieux et cela est vrai car mes hommes se comptent par millions. [...]

Le monde m'a oublié, peu m'importe.

Je choisirai le jour où me rappeler à sa mémoire.

Ce sera mon triomphe et les tours colosses de New York se cabreront sous la puissance de mon rire.

En attendant ce jour, de ces hommes et de ces femmes qui n'ont rien et ne sont rien, je suis le témoin.

Onysos est celui qui les aime.

Je n'ai ni or ni repos à leur offrir, juste le partage du regard émigré.

J'embrasse cette foule de parias et je connaîtrai bientôt le visage de chacun d'entre eux (Gaudé, 2000 : 46-47).

La ville américaine occupe une place importante dans Onysos le furieux: Gaudé crée de New York - à travers de très beaux passages sur la mégalopole personnifiée ${ }^{23}$ - une mise en scène parfaite pour le métissage de nombreux mythèmes, ainsi qu'un cadre presque cinématographique pour son premier texte théâtral.

\footnotetext{
${ }^{23}$ Pendant que New York "se frotte les yeux et se secoue à l'arête dorsale comme un cachalot d'asphalte et de verre qui s'ébroue avant la première plongée », les machines à café « comme des dragons d'acier, expulsent des nuages de vapeur brûlante » (Gaudé, $2000: 45)$.
} 


\section{CONCLUSION}

Contrairement aux représentations de Dionysos dans la mythologie grecque, Onysos n'est plus un dieu intouchable, mais un demi-dieu qui finit dans le caniveau de l'existence humaine moderne. Après une vie remplie de violences et de plaisirs charnels, Onysos trouve sa place et sa rédemption auprès des hommes qui n'ont rien. Bien qu'il incarne de nombreuses caractéristiques dionysiaques, Onysos est, avant tout, porteur de caractéristiques humaines : fidèle, vulnérable, rebelle et intrépide. Né pour la deuxième fois à partir de son cœur, il pleure, il souffre, il vieillit et il aime comme les hommes. Tandis que sa fureur est une réponse aux injustices humaines à travers les siècles, son choix à rester auprès des hommes, malgré leurs défauts, est une leçon pour l'homme contemporain qui, semble-t-il, vit le culte dionysiaque dans un contexte négatif.

Pour s'approprier le mythe dionysiaque sans le fardeau de ses nombreuses variantes précédentes, Gaudé choisit, relie et transforme « les plus petites unités de discours mythiquement significatives » qui lui permettent de tisser sa propre antiquité imaginaire auxquels il ajoute ensuite les mythèmes originels, auctoriaux. Le choix des mythèmes que nous avons individué, en nous appuyant sur la mythocritique de Philippe Walter, démontre l'intention de l'auteur de croiser, dans son premier texte théâtral, ses deux idées principales : illustrer le côté humain du dieu antique et mettre en lumière la condition humaine moderne, en rapprochant Dionysos de l'homme contemporain. Tandis que le métissage des mythèmes hétérogènes réactualise le mythe dyonisiaque, l'ambigüité du personnage mythique complexe aide l'écrivain à explorer ses faiblesses et créer - par la puissance suggestive des mots - un héros plus humain que divin, ce qui est la plus grande force d'Onysos le furieux. 
Nebojša Vlaškalić

DIONYSUS IN NEW YORK: FRAGMENTS OF THE DIONYSIAN MYTH IN ONYSOS LE FURIEUX BY LAURENT GAUDÉ

\section{Summary}

Onysos le furieux is the first play written by contemporary French writer Laurent Gaudé. Written in 1997 and published in 2000, this epic monologue offers a new reading of the Dionysian myth. Sitting on the platform of a New York City subway, Onysos speaks and takes us on a journey through time and through his story, which is, in reality, that of Dionysus - a little less of a god, a little more of a man. The writer's intention is not to rewrite or adapt one of the many classical variations of the Dionysian myth, but to weave a new mythological space, his own imaginary antiquity, from heterogeneous and amalgamated mythical particles. Based on Philippe Walter's mythocritique, in this work we will analyze the mythemes, the smallest mythically significant units of narration, which constitute the puzzle of the myth in Onysos le furieux. We will try to retrace the borrowed mythemes from the different classical variants of the myth of Dionysus (the tragic birth, the dismemberment, the descent into Hell, the episode with Proscumnos, Dionysus transvestite, etc.), as well as the constructed mythems, written by the author.

Key words: Dionysian myth, Onysos le furieux, mythemes, mythocritique, Laurent Gaudé, contemporary French literature.

\section{RÉFÉRENCES BIBLIOGRAPHIQUES}

Augé-Rabier, C. (2013). Méduse à trois têtes: de la polyphonie au tragique. Médée Kali de Laurent Gaudé. MuseMedusa, 1. Disponible sur http://musemedusa.com/dossier_1/auge-rabier/

Brunel, P. (2000). Dictionnaire des Mythes Littéraires. Paris: Éditions du Rocher.

Brunel, P. (1992). Mythocritique, Théorie et parcours. Paris : PUF.

Chauvin, D.-Siganos, A. \& Walter, Ph. (2005). Questions de mythocritique, Dictionnaire. Paris : Éditions Imago.

Detienne, M. (1981). L'Invention de la mythologie. Paris : Gallimard.

Durand, G. (1992). Figures mythiques et visages de l'œuvre: de la mythocritique à la mythanalyse. Paris : Berg international, Dunod.

Durand, G.-Chauvin, D. (éd.) (1996). Champs de l'Imaginaire. Grenoble : Ellug.

Eliade, M. (1963). Aspects du mythe. Paris : Gallimard. 
Euripide. (2005). Les Bacchantes. Traduction Jean et Mayotte Bollack. Paris : Les Éditions de Minuit.

Gaudé, L. (2000). Onysos le furieux. Paris : Actes Sud-Papiers.

Heidmann, U. (2010). Mythes, littératures, cultures. Quelles méthodes pour comparer les (re)configurations des mythes gréco-romains? Lausanne : CLE-UNIL.

Lacarrière, J. (1998). Au cœur des mythologies. Paris : Gallimard, Collection Folio.

Onysos le furieux de Laurent Gaudé. Notes de mise en scène par Bruno Ladet. Théâtre contemporain. Consulté le 29 janvier 2021, disponible sur http://www.theatre-contemporain.net/spectacles/Onysos-le-furieux8003/ensavoirplus/idcontent/35061

Leonardy, E. (1994). Mythe et littérature. Louvain-La-Neuve : Collège Érasme/Éditions Nauwelaerts.

Moingeon, Ph. (2007). Introduction à la mythologie contemporaine. Groslay : Éditions Ivoire-Clair.

Rubino, G. (éd.) (2006). Voix du contemporain. Histoire, mémoire et réel dans le roman français d'aujourd'hui. Roma : Bulzoni.

Vernant, J.-P. (1980). Le Mythe au réfléchi. Le Temps de la réflexion, 1. Paris: Gallimard. 21-25.

Viart, D.-Vercier, B. \& Evrard, F. (2008). La littérature française au présent : héritage, modernité, mutations. Paris : Bordas.

Viart, D. (2013). Anthologie de la littérature contemporaine française : Romans et récits depuis 1980. Paris : Armand Collin.

Vlaškalić, N. (2020). Mitska slagalica: ukršteni mitovi u delu Medeja Kali Lorana Godea. In: Gudurić, S.-Radić-Bojanić, B. \& Mutavdžić, P. (éd.) (2020). Jezici i kulture u vremenu i prostoru, IX/2. Novi Sad : Filozofski fakultet. 39-47.

Walter, Ph. (2008). La Fée Mélusine. Le Serpent et l'oiseau. Paris : Imago. 